\title{
Prediction early recurrence of hepatocellular carcinoma eligible for curative ablation using a Radiomics nomogram
}

Chunwang Yuan ${ }^{1,2+}$, Zhenchang Wang ${ }^{1 *}$, Dongsheng $\mathrm{Gu}^{3+}$, Jie Tian ${ }^{3,5,6,7^{*}}$, Peng Zhao ${ }^{2}$, Jingwei Wei ${ }^{3}$, Xiaozhen Yang ${ }^{2}$, Xiaohan $\mathrm{HaO}^{3}$, Di Dong ${ }^{3}$, Ning He${ }^{2}$, Yu Sun ${ }^{2}$, Wenfeng $\mathrm{Gao}^{2}$ and Jiliang Feng ${ }^{4}$

\begin{abstract}
Background: Predicting early recurrence (ER) after radical therapy for HCC patients is critical for the decision of subsequent follow-up and treatment. Radiomic features derived from the medical imaging show great potential to predict prognosis. Here we aim to develop and validate a radiomics nomogram that could predict ER after curative ablation.

Methods: Total 184 HCC patients treated from August 2007 to August 2014 were included in the study and were divided into the training $(n=129)$ and validation $(n=55)$ cohorts randomly. The endpoint was recurrence free survival (RFS). A set of 647 radiomics features were extracted from the 3 phases contrast enhanced computed tomography (CECT) images. The minimum redundancy maximum relevance algorithm (MRMRA) was used for feature selection. The least absolute shrinkage and selection operator (LASSO) Cox regression model was used to build a radiomics signature. Recurrence prediction models were built using clinicopathological factors and radiomics signature, and a prognostic nomogram was developed and validated by calibration.

Results: Among the four radiomics models, the portal venous phase model obtained the best performance in the validation subgroup ( $\mathrm{C}$-index $=0.736$ ( $95 \% \mathrm{Cl}: 0.726-0.856)$ ). When adding the clinicopathological factors to the models, the portal venous phase combined model also yielded the best predictive performance for training (Cindex $=0.792(95 \% \mathrm{Cl}: 0.727-0.857)$ and validation (C-index $=0.755(95 \% \mathrm{Cl}: 0.651-0.860)$ subgroup. The combined model indicated a more distinct improvement of predictive power than the simple clinical model (ANOVA, $P<0$. 0001).

Conclusions: This study successfully built a radiomics nomogram that integrated clinicopathological and radiomics features, which can be potentially used to predict ER after curative ablation for HCC patients.
\end{abstract}

Keywords: Hepatocellular carcinoma, Radiomics, Recurrence, forecasting, Ablation techniques

\footnotetext{
*Correspondence: cjr.wzhch@vip.163.com; tian@ieee.org

Chunwang Yuan and Dongsheng Gu are co-first author.

'Department of Radiology, Beijing Friendship Hospital, Capital Medical

University, No.95, Yong An Road, Xicheng District, Beijing 100050, China

${ }^{3}$ Key Laboratory of Molecular Imaging, Institute of Automation, Chinese

Academy of Sciences, No.95 Zhongguancun East Road, Haidian District,

Beijing 100190, China

Full list of author information is available at the end of the article
}

(c) The Author(s). 2019 Open Access This article is distributed under the terms of the Creative Commons Attribution 4.0 International License (http://creativecommons.org/licenses/by/4.0/), which permits unrestricted use, distribution, and reproduction in any medium, provided you give appropriate credit to the original author(s) and the source, provide a link to the Creative Commons license, and indicate if changes were made. The Creative Commons Public Domain Dedication waiver (http://creativecommons.org/publicdomain/zero/1.0/) applies to the data made available in this article, unless otherwise stated. 


\section{Background}

Hepatocellular carcinoma (HCC) is the fifth most common cancer and the third leading cause of cancer-related death globally [1], In China, it ranks fourth and second respectively [2]. Surgical resection and liver transplantation are standard treatments for early HCC, but their applications are limited because the severe liver dysfunction, concomitant diseases and the shortage of liver grafts. Therefore, several non-surgical local ablation techniques have been introduced, such as radiofrequency ablation (RFA), microwave ablation (MWA), and cryoablation (Cryo-A) [3]. These methods are currently widely used because they are simple, safe, effective, minimally invasive, repeatable, and require a short hospital stay. Hence, they are often considered the best options for $\mathrm{HCC}$ patients with Barcelona Clinic Liver Cancer (BCLC) stage 0-A and some selected BCLC stage B (Combined with transcatheter arterial chemoembolization (TACE) ) [4-7], who are not suitable for resection or liver transplantation.

ER after ablation (Intrahepatic new lesion occurs within 2 years after ablation) is one of the main factors of mortality [8-12].Patients with late recurrence (Intrahepatic new lesion occurs after 2 years post-ablation) could have a better survival than patients with ER [11, 12]. Therefore, early detection and timely treatment of HCC recurrence should improve prognosis [13], but up to now there is no really powerful tool for predicting ER after ablation.

A number of prognostic scoring systems and nomograms were developed to predict the risk of HCC recurrence after radical resection [14-16]. These systems are based on demographic, clinical, and biochemical factors that may be associated with tumor recurrence. CECT imaging can provide information and enhancement features of the entire tumor, and has been reportedly used to achieve $78 \%$ reconstruction of the global HCC gene-expression profiles by the combination of 28 morphologic imaging traits [17], thus making it possible to infer the biological behavior of the tumor through the imaging features [18-20]. Radiomics extracts large amounts of quantitative features from medical images to reveal disease characteristics that fail to be detected by the naked eye $[18,19,21-24]$. Radiomics features specific to each patient provide valuable information for personalized medicine [23]. In this light, radiomics could improve the prediction of HCC ER. A recent study showed that radiomics signatures were predictive for HCC ER after surgical resection [25].We want to develop and validate radiomics signatures that could predict RFS after curative ablation in order to actively adopt tailored follow-up strategies and interventions.

\section{Methods}

\section{Patients and laboratory, pathology, imaging data}

The retrospective study was approved by institution ethics committee, which enrolled 184 HCC patients hospitalized from August 2007 to August 2014 and gained curative ablation. All ablation procedures were performed by CT-guided percutaneous route, which were performed by Interventional radiologists with more than 500 ablation procedures experience. Patients were randomly divided into training cohort $(n=129)$ and validation cohort $(n=55)$. The human experimentation guidelines of the PRC were followed, the informed consent was not required. All patients were diagnosed with HCC by percutaneous biopsy pretherapy. The CECT images were entirely from a 64-row spiral CT system (Lightspeed VCT, GE Healthcare, Pittsburgh, PA, USA), with format of digital imaging and communications in medicine (DICOM). The pathological data were obtained totally from of hospital pathology center. Figure 1 shows the patient flowchart. The inclusion criteria: 1) 18-75 years of age; 2) CECT was performed within 2 weeks before ablation; 3) BCLC stage A to B2; 4) unwilling to undergo hepatectomy or liver transplantation; 5) well-preserved liver function, i.e., Child-Pugh class A/B, serum total bilirubin level $\leq 3 \mathrm{mg} / \mathrm{dl}$; and 6) eastern cooperative oncology group (ECOG) performance status score $\leq 2$. The exclusion criteria: 1 ) tumor thrombus in a major hepatic vessel; 2) extrahepatic metastases; 3) uncontrollable ascites, history of hepatic encephalopathy, or variceal bleeding occurred less than 1 month; 4) Child-Pugh class C; 5) severe coagulation disorder (platelet count less than $5 \times 10^{3} / \mu$ Lor prothrombin activity $<50 \%$; 6) history of secondary malignancy; 7) severe dysfunction of the heart, brain, kidney, or other organs; 8) active infection (except viral hepatitis); or 9) refusal of ablation. Follow-up tactics: All patients underwent liver CECT/CEMRI scan, test of liver function and tumor markers (such as AFP) every 3 months after ablation. The shortest follow-up time was $>3$ years. Tumor recurrence was diagnosed by CECT/CEMRI showing arterial hyperenhancement and wash out appearance in the portal venous phase [26].The baseline characteristics of enrolled patients were listed in Table 1.

\section{Statistical analysis}

Individual variables were analyzed for significant differences in the training and validate cohort using the Mann Whitney $\mathrm{U}$ test for continuous variables and $\mathrm{x}^{2}$ test for categorical variables. All statistical analyses were implemented using PASW Statistics 18.0.0 and $\mathrm{R}$ (version 3.4.1). P-values (Two-sided) $<0.05$ were regarded as statistically significant. 


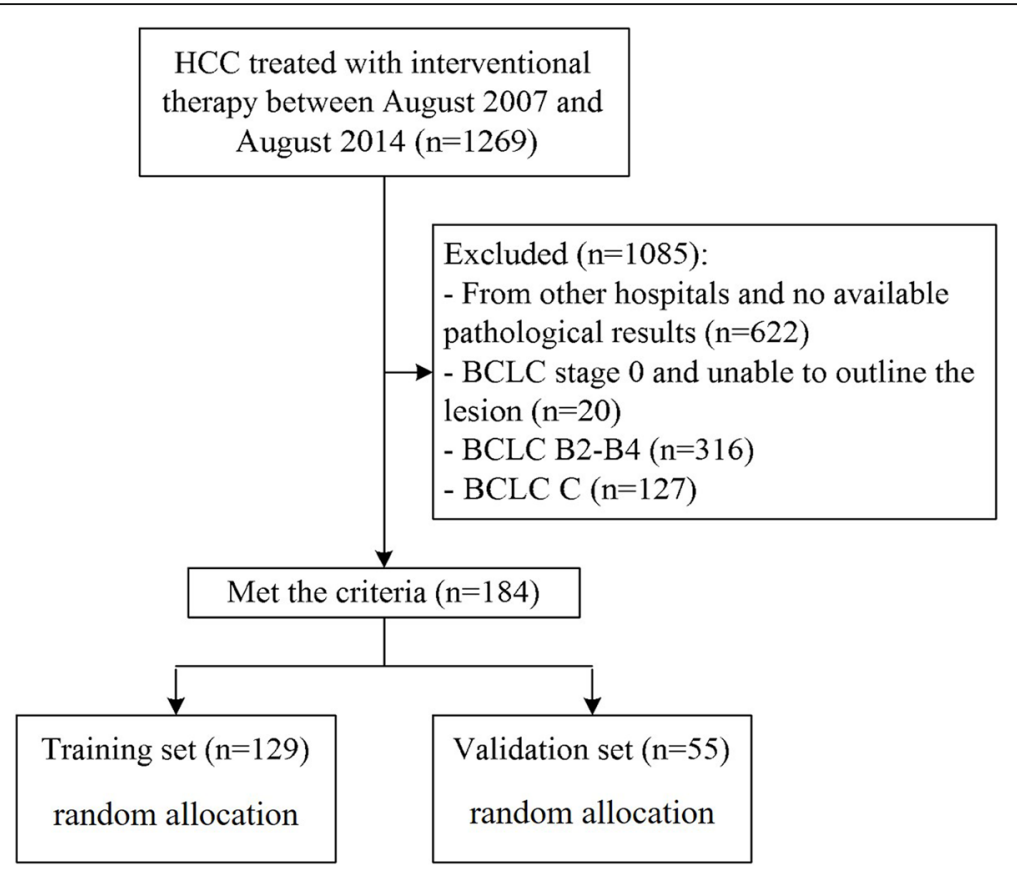

Fig. 1 Patient flowchart

Tumor segmentation, radiomics feature extraction, Clinicopathological factors analysis, clinical model building, Radiomics feature selection, Radiomics signature building and model evaluation Tumor segmentation

Three-dimensional manual segmentation of tumors were performed by a radiologist with more than $15 \mathrm{yrs}$. work experience, using the ITK-SNAP software (http://www. itksnap.org/pmwiki/pmwiki.php). Regions of interest (ROI) were drawn on the images from the arterial, portal venous, and parenchymal phases, slice by slice, for each patient $[25,27]$. The final segmentation results were validated by a senior radiologist with more than 20 yrs. work experience to ensure segmentation validity. Test-retest datasets were obtained to test the reproducibility of the extracted features in 20 patients randomly selected for repeated segmentation by the senior radiologist.

\section{Radiomics feature extraction}

A set of 647 radiomics features that reflected the machine-read radiological characteristics and subtle textural information was extracted from the segmented ROIs. Image filtering was implemented on original 3-dimension tumor slices with undecimated wavelet transform [27], which decomposed the original image into eight decompositions. Features were extracted from both the original and filtered images and could be divided into two types: non-textural features and textural features. The non-textural features included shape, size, and intensity features. Shape and size features captured the direct-viewing characteristics of the lesion. Intensity features depicted the characteristics of the histogram of the tumor lesion. Textural features were extracted based on four textural matrixes: Gray Level Co-occurrence Matrix (GLCM), Gray Level Run-Length Matrix (GLRLM), Gray Level Size Zone Matrix (GLSZM), and Neighborhood Gray-Tone Difference Matrix (NGTDM) [28-30]. All features' types and names are presented in Additional file 1: Table S2. Features extraction was performed using Matlab 2014a (MathWorks, Natick, MA, USA).

\section{Clinicopathological factors and clinical model building}

Clinicopathological factors with $P$-values $<0.10$ in univariable Cox proportional hazard regression analysis were integrated into a stepwise multivariable Cox model. Variables with P-values $<0.05$ in the multivariable analysis were identified as potential clinicopathological factors related to RFS and were included for the clinical model building.

\section{Radiomics feature selection}

The intra-class correlation coefficient (ICC) was calculated to determine the stability of the features, followed by the test-retest setting. Features with an ICC $<0.75$ were excluded from the final feature set. In order to reduce the redundancy and the unnecessary complexity for computation and modeling, MRMRA was used for feature selection. The aim of this algorithm was to select a feature subset which can achieve the best characterization of the difference between the two targeted classified groups, 
Table 1 Patient characteristics in the training and validation datasets

\begin{tabular}{|c|c|c|c|}
\hline Characteristics & $\begin{array}{l}\text { Training dataset } \\
(N=129)\end{array}$ & $\begin{array}{l}\text { Validation dataset } \\
(N=55)\end{array}$ & $P$ \\
\hline $\mathrm{Age}^{\mathrm{a}}$, median $(\mathrm{IQR})$, years & $57(49-63)$ & $57(51-61)$ & 0.652 \\
\hline Gender, n (\%) & & & 0.673 \\
\hline Male & 95 (73.6) & $41(74.5)$ & \\
\hline Female & $34(26.4)$ & $14(25.5)$ & \\
\hline Tumor diameter ${ }^{\mathrm{a}}$, median (IQR), cm & $3.3(2.5-4.7)$ & $3.5(2.7-5.2)$ & 0.384 \\
\hline Histological grade, n (\%) & & & 0.086 \\
\hline I & $31(24.0)$ & $16(29.1)$ & \\
\hline$\|$ & $66(51.2)$ & $32(58.2)$ & \\
\hline III & $32(24.8)$ & $7(12.7)$ & \\
\hline $\mathrm{AFP}^{\mathrm{a}}$, median $(\mathrm{IQR})$ & $14.2(3.6-144.3)$ & $20.5(4.9-132.3)$ & 0.128 \\
\hline Cause, n (\%) & & & 0.467 \\
\hline Alcohol & $7(5.4)$ & $4(7.3)$ & \\
\hline $\mathrm{HCV}$ & $14(10.9)$ & $6(10.9)$ & \\
\hline HBV & $106(82.2)$ & $45(81.8)$ & \\
\hline $\mathrm{HBV}+\mathrm{HCV}$ & $2(1.6)$ & 0 & \\
\hline ECOG, n (\%) & & & 0.614 \\
\hline 0 & $3(2.3)$ & 0 & \\
\hline 1 & $126(97.7)$ & $55(100)$ & \\
\hline CK19+, n (\%) & & & 0.633 \\
\hline 0 & $107(82.9)$ & $46(83.6)$ & \\
\hline 1 & $17(13.2)$ & $7(12.7)$ & \\
\hline 2 & $1(0.8)$ & $1(1.8)$ & \\
\hline 3 & $4(3.1)$ & $1(1.8)$ & \\
\hline GPC3+, n (\%) & & & 0.833 \\
\hline 0 & $40(31)$ & $13(23.6)$ & \\
\hline 1 & $47(36.4)$ & $22(40)$ & \\
\hline 3 & 25 (19.4) & $9(16.4)$ & \\
\hline 4 & $17(13.2)$ & $11(20)$ & \\
\hline $\mathrm{HBsAg}+, \mathrm{n}(\%)$ & & & 0.960 \\
\hline 0 & 74 (57.4) & $33(60)$ & \\
\hline 1 & $38(29.5)$ & $20(36.4)$ & \\
\hline 2 & $12(9.3)$ & $2(3.6)$ & \\
\hline 3 & $5(3.9)$ & 0 & \\
\hline $\mathrm{HBCAg}+, \mathrm{n}(\%)$ & & & 0.328 \\
\hline 0 & $121(93.8)$ & $48(87.3)$ & \\
\hline 1 & $7(5.4)$ & $7(12.7)$ & \\
\hline 2 & $1(0.8)$ & 0 & \\
\hline Child-Pugh, n (\%) & & & 0.102 \\
\hline 0 & $122(94.6)$ & $55(100)$ & \\
\hline 1 & $7(5.4)$ & 0 & \\
\hline BCLC, n (\%) & & & 0.382 \\
\hline A & $81(62.8)$ & $27(49.1)$ & \\
\hline B1 & $48(37.2)$ & $28(50.9)$ & \\
\hline
\end{tabular}


Table 1 Patient characteristics in the training and validation datasets (Continued)

\begin{tabular}{lll}
\hline Characteristics & $\begin{array}{l}\text { Training dataset } \\
(N=129)\end{array}$ & $\begin{array}{l}\text { Validation dataset } \\
(N=55)\end{array}$ \\
\hline Ablation approach & & \\
RFA & $86(66.7)$ & $39(70.9)$ \\
MWA & $27(20.9)$ & $14(25.5)$ \\
CRYO-A & $16(12.4)$ & $2(3.6)$ \\
RFS time ${ }^{a}$, median (IQR), months & $15(6-30)$ & $13(5-36)$
\end{tabular}

Note: Data are shown as number of patients, with the percentage in parentheses unless noted. No significant differences were found between the training cohort and the validation datasets

aData are medians, with interquartile ranges in parentheses

considering the restriction that these features were mutually dissimilar with each other to the full extent, but marginally related to the selected clinical outcome [31]. Moreover, MRMRA was proved to be more stable for the feature extraction process, especially for radiomics [32]. A set of 20 potential features were selected for further model construction according to the output score of each feature by MRMRA. The "irr" R package was used for computation of intra-class correlation coefficient. The "mRMRe" $\mathrm{R}$ package was used for MRMRA feature selection.

\section{Radiomics signature building}

We used the extensive LASSO method for further variable selection in Cox proportional hazards model with the 20 selected features from the training dataset [33, 34]. We chose the optimal feature set that had the maximal cross-validation log partial likelihood. Non-zero coefficients were defined as the weight for each selected feature, which indicated the hazard ratio between the feature and survival. Each patient's radiomics signature was generated by multiplying the selected features with their respective coefficients. We utilized the LASSO Cox regression to build prediction models on the training dataset and validated their predictive effectiveness using the validation cohort. The prognostic value of the radiomics signature was estimated by Kaplan-Meier (KM) curves. Patients were stratified into the high and low risk groups through the median value of radiomics signature (arterial phase: 3.037; portal venous phase: 2.687, and parenchymal phase: 2.493 ). We compared the two KM survival curves by the log-rank test. The glmnet $\mathrm{R}$ package was used for LASSO Cox variable selection and model building. The survival $\mathrm{R}$ package was used for comparison of the survival curves.

\section{Model evaluation}

The final Cox proportional hazard model incorporated the clinicopathological factors along with three phases radiomics signatures. The performance of the clinical model, radiomics models, and the final combined model were evaluated by the concordance index (C-Index) [27] with 1000 bootstrap resamples, which is the area under the curve for continuous time-to-event survival data and can measure the discrimination of a prognostic model by the area under the curve of continuous time-to-event survival data. A value of 1 indicates perfect discrimination and 0.5 represents discriminative power equal to randomness. The Hosmer-Leme show test was applied for the prediction model [35]. We further built a nomogram for the model to provide a more direct way to determine the 1-, 2- and 3-year RFS rates. A calibration curve was plotted to analyze the prognostic performance of the nomogram on both the training and validation datasets [36]. The "rms" R package was used for Cox proportional hazards regression, nomograms, and calibration curves.

\section{Results}

1. Demographic data are provided in Table 1.

2. The results of uni- and multivariable analyses for the clinical model construction are showed in Table 2.

3. Radiomics feature selection and model construction.

Presented in Fig. 2. The top 20 features were adopted as inputs to the LASSO Cox model [34, 35]. According to the leave-one-out cross validation, the final numbers of features included were 5,5 , and 10 for the radiomics model construction in the arterial, portal venous, and parenchymal phases, respectively. The selected features are shown in supporting information (Additional file 1: Table S1), and the detailed information is presented in supporting information (Additional file 1: Table S2). The corresponding formulas of the radiomics signature for the three phases are separately shown in supporting information (Additional file 1: Table S3). Univariate Cox proportional hazard model was built with a single radiomics signature from the three phases CECT images respectively, and multivariable Cox proportional hazard model integrated the three phases radiomics signatures was built as a fusion model. 
Table 2 Results of the univariable and multivariable analyses

\begin{tabular}{|c|c|c|c|c|}
\hline \multirow[t]{2}{*}{ Clinical predictors } & \multicolumn{2}{|c|}{ Univariable } & \multicolumn{2}{|c|}{ Multivariable } \\
\hline & $P$ & HR $(95 \% \mathrm{Cl})$ & $P$ & HR $(95 \% \mathrm{Cl})$ \\
\hline Gender & 0.57 & $1.159(0.697-1.927)$ & & \\
\hline Age & 0.527 & $1.004(0.992-1.016)$ & & \\
\hline Tumor maximal diameter & 0.59 & $0.975(0.888-1.07)$ & & \\
\hline Grade & 0.099 & $0.791(0.599-1.045)$ & 0.3442 & $0.889(0.696-1.135)$ \\
\hline AFP & 0.932 & $1(1-1)$ & & \\
\hline Etiology & 0.28 & $0.823(0.577-1.172)$ & & \\
\hline ECOG & 0.129 & $4.798(0.634-36.289)$ & & \\
\hline CK19+ & 0.46 & $1.127(0.821-1.545)$ & & \\
\hline GPC3+ & 0.251 & $1.134(0.915-1.406)$ & & \\
\hline HBsAg+ & 0.714 & $1.054(0.797-1.393)$ & & \\
\hline $\mathrm{HBCAg}+$ & 0.713 & $0.856(0.375-1.955)$ & & \\
\hline Child-Pugh & 0.087 & $2.128(0.897-5.051)$ & $0.007^{*}$ & $2.762(1.317-5.791)$ \\
\hline $\mathrm{BCLC}$ & $<0.001$ & $2.145(1.55-2.967)$ & $<0.001^{*}$ & $4.834(2.698-8.662)$ \\
\hline
\end{tabular}

Note: ${ }^{*} P<0.05$

4. Validation of the radiomics signature

Each single radiomics signature satisfied the discriminative power in the univariable Cox model. The arterial, portal venous, parenchymal phase signatures yielded $\mathrm{HR}$ of $11.46(P<0.0001,95 \% \mathrm{CI}$ : $4.14-31.68), \quad 20.00(P=0.0002,95 \% \mathrm{CI}: 4.14-96.61)$, $6.16(\mathrm{P}<0.0001,95 \% \mathrm{CI}: 3.35-11.34)$, respectively. KM curves for all phases on both the training and validation datasets were developed (Fig. 3). The log-rank test revealed a significant difference $(P<$ 0.001 ) between the high- and low-risk subgroups for all phases.

\section{Performance of the different models}

A validation dataset with 55 patients included randomly was used to evaluate the models' predictive power. One clinical model, four radiomics models, and four combined models were built. The clinical model had the worst predictive performance in the training dataset with a $\mathrm{C}$-index of 0.649 (95\%CI: $0.592-0.706)$ and the validation dataset with a C-index of 0.556 (95\%CI: 0.471-0.641). Among the four radiomics models, the fusion model with three phases radiomics signatures had the best predictive performance for RFS, with a C-index of 0.791 (95\%CI: 0.726-0.856) in the training dataset, but did not show the best performance
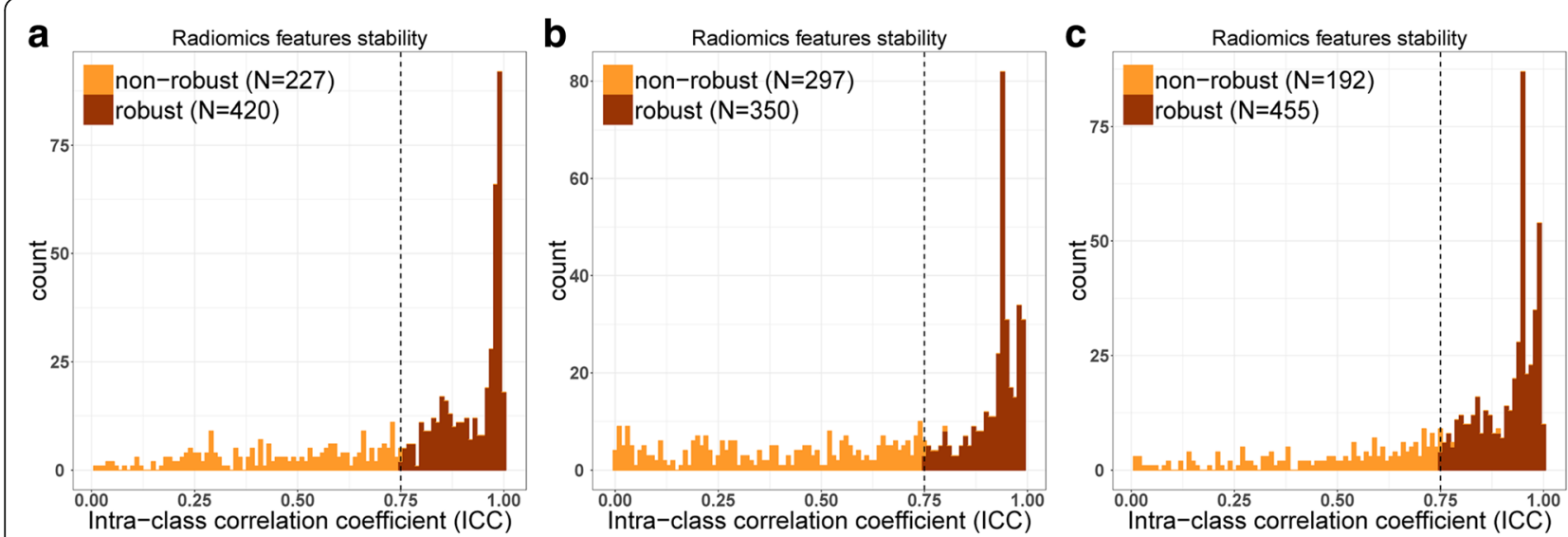

Fig. 2 Histogram of the intra-class correlation coefficient (ICC). For the 20 random selected patients from the overall dataset, we extracted the radiomics features from the test and re-test scans. The ICC was used to determine the stability of the features. Features with an ICC $<0.75$ were excluded from the analysis. After robustness test, 420 of the initial 647 CT image features in the arterial phase, 350 in the portal venous phase, and 455 in the parenchymal phase were retained 
a

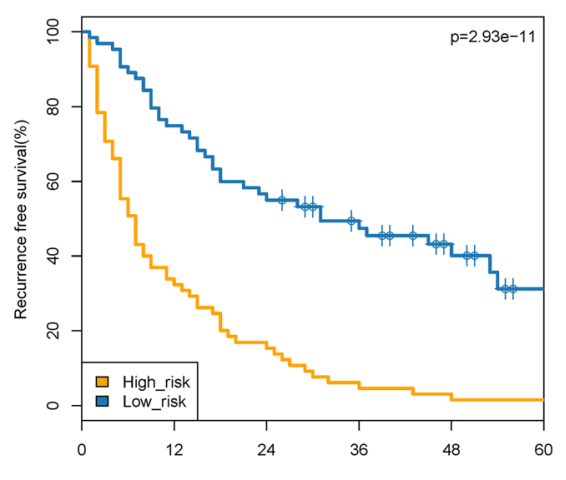

$\begin{array}{llllllllllll}\text { High_risk } 65 & 36 & 22 & 16 & 11 & 6 & 4 & 3 & 2 & 1 & 1\end{array}$
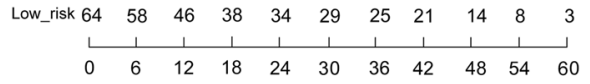

C

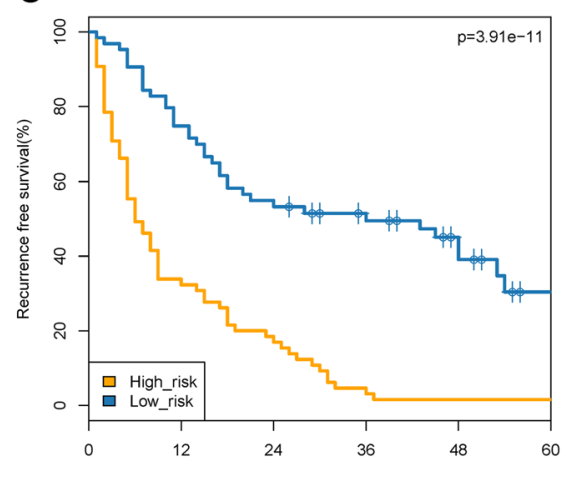

Time Since Diagnosis(months)

High_risk65 $36 \begin{array}{llllllllll}62 & 22 & 17 & 12 & 7 & 3 & 1 & 1 & 1 & 1\end{array}$ $\begin{array}{lllllllllll}\text { Low_risk } 64 & 58 & 46 & 37 & 33 & 28 & 26 & 23 & 15 & 8 & 3\end{array}$

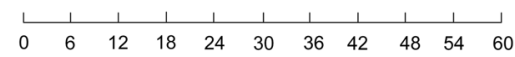

e

Survival Distributions in training dataset

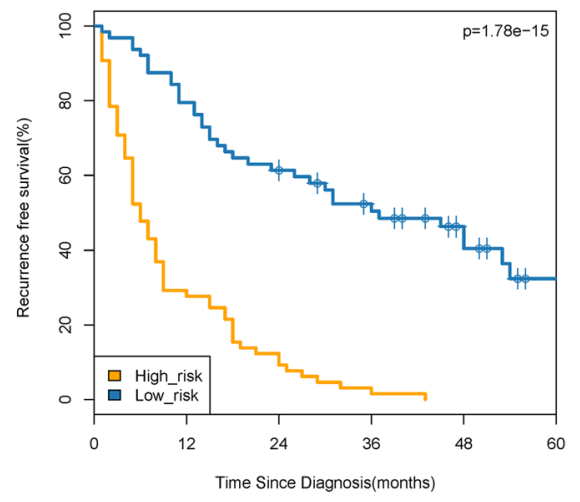

High_risk65 $34 \quad \begin{array}{lllllllll}34 & 14 & 8 & 3 & 2 & 0 & 0 & 0 & 0\end{array}$

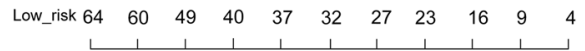

b Survival Distributions in validation dataset

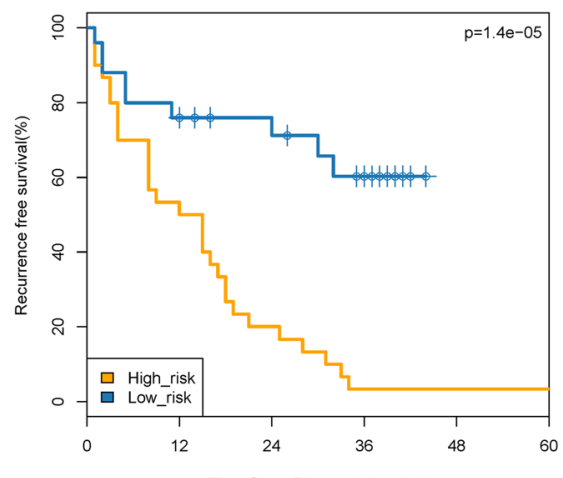

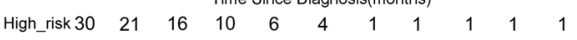

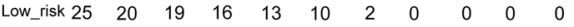

$\begin{array}{lcccccccccc} & 1 & 1 & 1 & 1 & 1 & 1 & 1 & 1 & 1 & \\ 0 & 6 & 12 & 18 & 24 & 30 & 36 & 42 & 48 & 54 & 60\end{array}$

d Survival Distributions in validation dataset

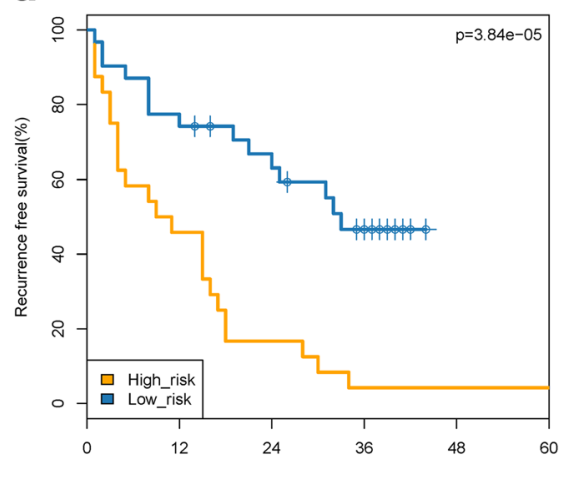

Time Since Diagnosis(months)

$\begin{array}{lllllllllll}\text { High_risk 24 } & 14 & 11 & 6 & 4 & 3 & 1 & 1 & 1 & 1 & 1\end{array}$

$\begin{array}{lllllllllll}\text { Low_risk } 31 & 27 & 24 & 20 & 18 & 14 & 10 & 2 & 0 & 0 & 0\end{array}$

$\begin{array}{ccccccccccc} & 1 & 1 & \mid & 1 & 1 & \mid & \mid & 1 & 1 & \\ 0 & 6 & 12 & 18 & 24 & 30 & 36 & 42 & 48 & 54 & 60\end{array}$

f Survival Distributions in validation dataset

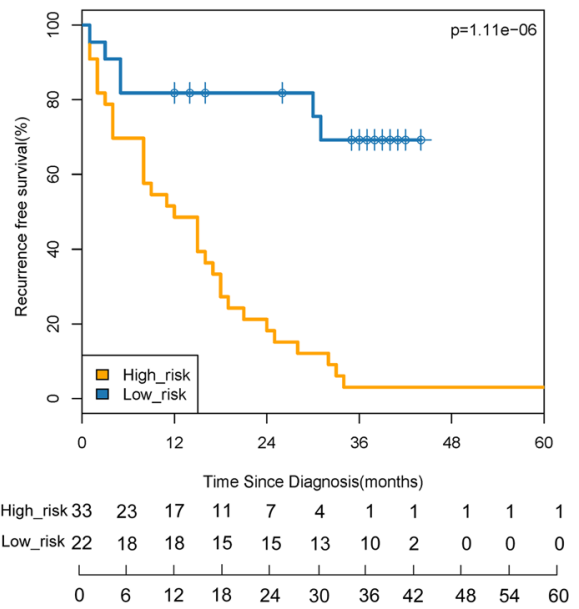

Fig. 3 Kaplan-Meier analyses of recurrence-free survival based on the proposed signature with cut-off values as the median of the training dataset. a Training dataset in the arterial phase. b Validation dataset in the arterial phase. c Training dataset in the portal venous phase. $\mathbf{d}$ Validation dataset in the portal venous phase. e Training dataset in the parenchymal phase. $\mathbf{f}$ Validation dataset in the parenchymal phase 
in the validation dataset, with a C-index of 0.690 (95\%CI: $0.586-0.795)$. The portal venous phase radiomics model obtained the best performance in the validation dataset with a C-index of 0.736 (95\%CI: 0.726-0.856). When adding the clinicopathological factors to the four radiomics models, the combined model consisting of the portal venous phase radiomics signatures yielded the best predictive power in the validation dataset $(\mathrm{C}$-index $=0.755$ [95\%CI: 0.651-0.860]), which also indicated a distinct improvement for the clinical model with a significant difference (ANOVA, $P<0.0001$ ) (Table 3). RFS during follow-up and the 1-, 2- and 3-year recurrence rates in both subgroups of portal venous phase are listed in Table 4. Patients with low radiomics signature value (risk score) had better RFS. The mean RFS at 1-, 2- and 3-year showed significant difference between the both subgroups in the training dataset $(P=0.004, P<0.001$, $P=0.024)$, but in the validation dataset, there was significant difference between the two groups $(P=0.044)$ only for 3-year, for 1 - and 2-year no significant difference $(P=0.2169, P=0.3402)$.

\section{Nomogram construction and evaluation}

As the combined model incorporating the portal venous phase radiomics signature and the clinicopathological factor had the best predictive performance, we built a nomogram based on this final model (Fig. 4). Furthermore, calibration curves of the combined nomogram were plotted for the training and validation datasets (Fig. 5). The Hosmer-Leme show test of the model showed non-significant differences in the training $(P=$ $0.791)$ and validation $(P=0.471)$ datasets, which demonstrated a satisfying agreement.

Table 3 Predictive performance for RFS of the proposed models

\begin{tabular}{|c|c|c|}
\hline \multirow[t]{2}{*}{ Models } & \multirow{2}{*}{$\begin{array}{l}\text { Training dataset } \\
N=129 \\
\text { C-index }(95 \% \mathrm{Cl})\end{array}$} & \multirow{2}{*}{$\begin{array}{l}\text { Validation dataset } \\
N=55 \\
\text { C-index }(95 \% \mathrm{Cl})\end{array}$} \\
\hline & & \\
\hline \multicolumn{3}{|l|}{ Clinical model } \\
\hline clinicopathologic feature & $0.649(0.592-0.706)$ & $0.556(0.471-0.641)$ \\
\hline \multicolumn{3}{|l|}{ Radiomics model } \\
\hline Arterial phase & $0.767(0.702-0.832)$ & $0.694(0.623-0.832)$ \\
\hline Portal vein phase & $0.757(0.692-0.821)$ & $0.736(0.632-0.841)$ \\
\hline Parenchymal phase & $0.789(0.723-0.853)$ & $0.686(0.582-0.791)$ \\
\hline All phases & $0.791(0.726-0.856)$ & $0.690(0.586-0.795)$ \\
\hline \multicolumn{3}{|l|}{ Combined model } \\
\hline Arterial phase + Clinicopathologic feature & $0.797(0.732-0.862)$ & $0.732(0.628-0.837)$ \\
\hline Portal vein phase + Clinicopathologic feature & $0.792(0.727-0.857)$ & $0.755(0.651-0.860)$ \\
\hline Parenchymal phase + Clinicopathologic feature & $0.806(0.741-0.871)$ & $0.728(0.624-0.834)$ \\
\hline All phases + Clinicopathologic feature & $0.809(0.744-0.874)$ & $0.724(0.620-0.829)$ \\
\hline
\end{tabular}

\section{Discussion}

The use of radiomics in medicine is still in its infancy and additional studies are necessary to examine the radiomics signatures that could predict patient outcomes. To date, no study used radiomics to investigate the prediction of HCC recurrence after ablation. Therefore, this study aimed to develop and validate radiomics signatures that could predict RFS after curative ablation. A large number of radiomic features in our study were extracted, as it may cause overfitting compared with the number of cases, we performed feature reduction and selection for the final model building. Accordingly, the radiomics model constructed with optimal features subset achieved satisfying performance. Moreover, the results of nomogram indicated that this study successfully built a combined model that integrated clinicopathological factors and radiomics features. For BCLC stage 0-B2 HCC, ablation and surgery are among the preferred options. Especially, ablation can be conducted for patients who are not suitable for surgery [13]. These ablation techniques destroy tumor cells locally but the destroyed tumor is not removed from the body [37], which can release intact tumor proteins that may serve as some kind of vaccine against eventual HCC recurrence [38]. Therefore, the recurrence patterns and odds of ablations may be not the same as those of surgical resection. Studies that specifically examine the outcomes of ablation are therefore required.

The BCLC staging system has been endorsed and recommended by multiple authoritative academic organizations for prognosis and treatment stratification of HCC patients. Indeed, it includes patient-related factors, tumor extent and liver function, and an algorithmic component for management. The BCLC staging and Child-Pugh score are included in a number of models predicting $\mathrm{HCC}$ recurrence [38-40], they are also

-index (Harrell concordance index) indicates the predictive performance 
Table 4 RFS and recurrence rates in the high-risk and low-risk groups

\begin{tabular}{|c|c|c|c|c|c|c|}
\hline & \multicolumn{3}{|l|}{$\begin{array}{l}\text { Training Dataset } \\
N=129\end{array}$} & \multicolumn{3}{|c|}{ Validation Dataset $N=55$} \\
\hline & High-Risk Group & Low-Risk Group & $P$-value & High-Risk Group & Low-Risk Group & $P$-value \\
\hline No. of patients (\%) & $65(50.4)$ & $64(49.6)$ & & $24(43.6)$ & $31(56.4)$ & \\
\hline 1-year RFS Median, (IQR) & $4.5(2.0-6.25)$ & $7(5-10)$ & 0.004 & $4(2-5)$ & $8(2-8)$ & 0.2169 \\
\hline 2-year RFS Median, (IQR) & $5(2.25-9)$ & $11(7-16)$ & $<0.001$ & $6.5(3-15)$ & $10(5.75-15.5)$ & 0.3402 \\
\hline 3-year RFS Median, (IQR) & $6(3.0-16.0)$ & $13(7.5-19)$ & 0.024 & $9(3.5-16.5)$ & $17.5(8.0-26)$ & 0.044 \\
\hline \multicolumn{7}{|l|}{ No. of recurrences (\%) } \\
\hline At 1 year & $44(34.1)$ & $16(12.4)$ & & $13(23.6)$ & $9(14.5)$ & \\
\hline At 2 year & $54(41.9)$ & $29(22.5)$ & & $20(36.4)$ & $14(20.0)$ & \\
\hline At 3 year & 63 (48.8) & 39 (30.2) & & 23 (41.8) & $22(40.0)$ & \\
\hline
\end{tabular}

important factors associated RFS of HCC patients in our present study.

So far, one radiomics study of $\mathrm{HCC}$ examined the risk of ER after surgical resection. Indeed, Zhou et al. [25] developed a radiomics signature using 215 HCC of BCLC 0-C stage patients after hepatectomy to predict ER ( $<1$ year in their study), but not of 2 - and 3 -year recurrence. Because patients with $\mathrm{HCC}$ of BCLC C stage are not suitable for curative ablation, it was not included in our study. A number of authors assessed the biological behavior of HCC using imaging features [18-20]. Similarly, radiomics signatures were built for recurrence prediction of a variety of cancers after surgery [25, 41-44], but it has to be noted that ablation is usually not indicated in those cancers. The present study suggests that the combination of the portal venous phase radiomics signature and the clinicopathological data provides fair to good results in a technically homogeneous dataset, but to really establish a model like this, external validation and larger dataset would be required.

The present study has a number of strengths. First, all patients were diagnosed HCC by percutaneous biopsy before treatment. In addition, CT-guided percutaneous curative ablation (i.e., complete response according to

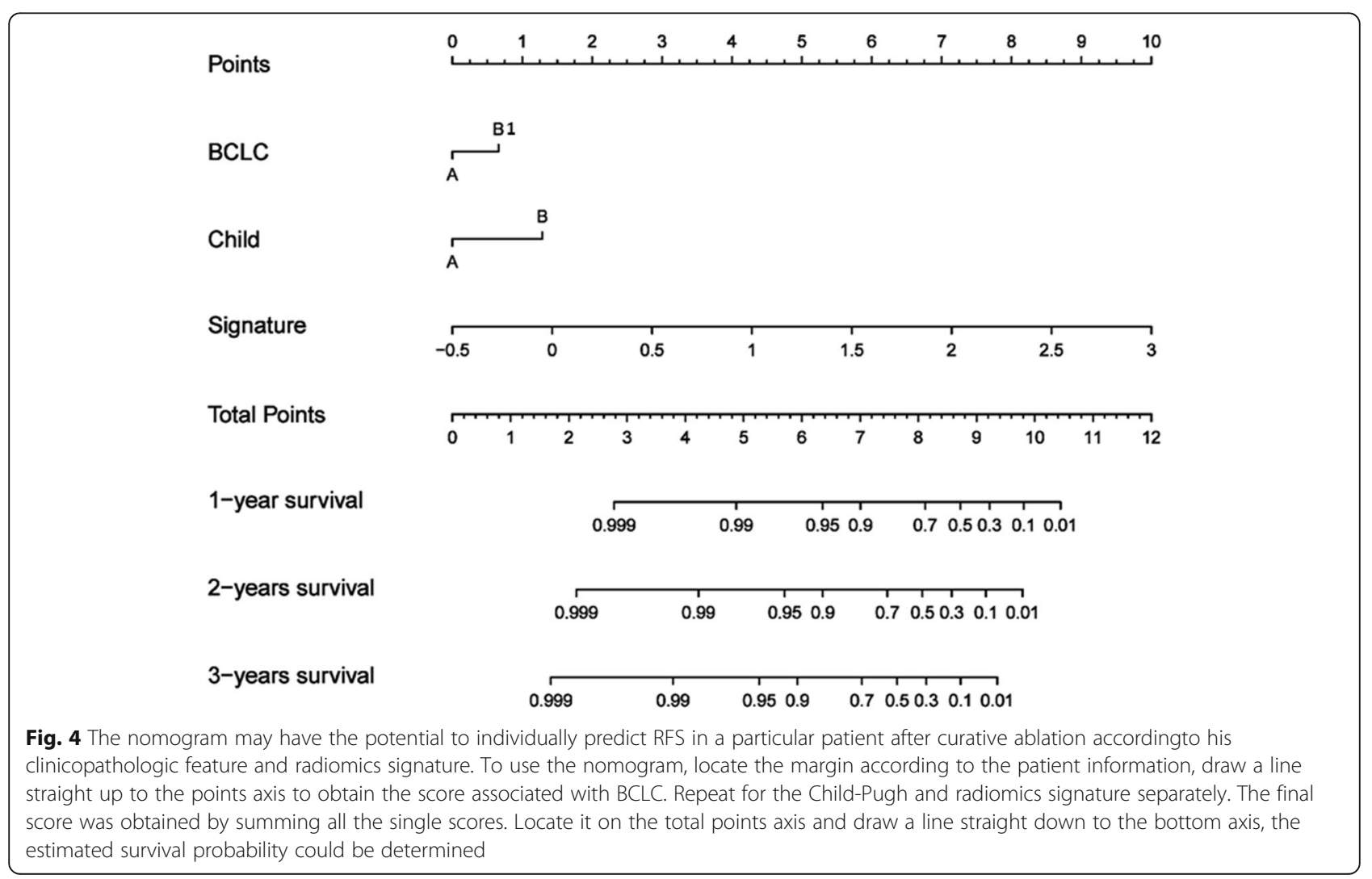



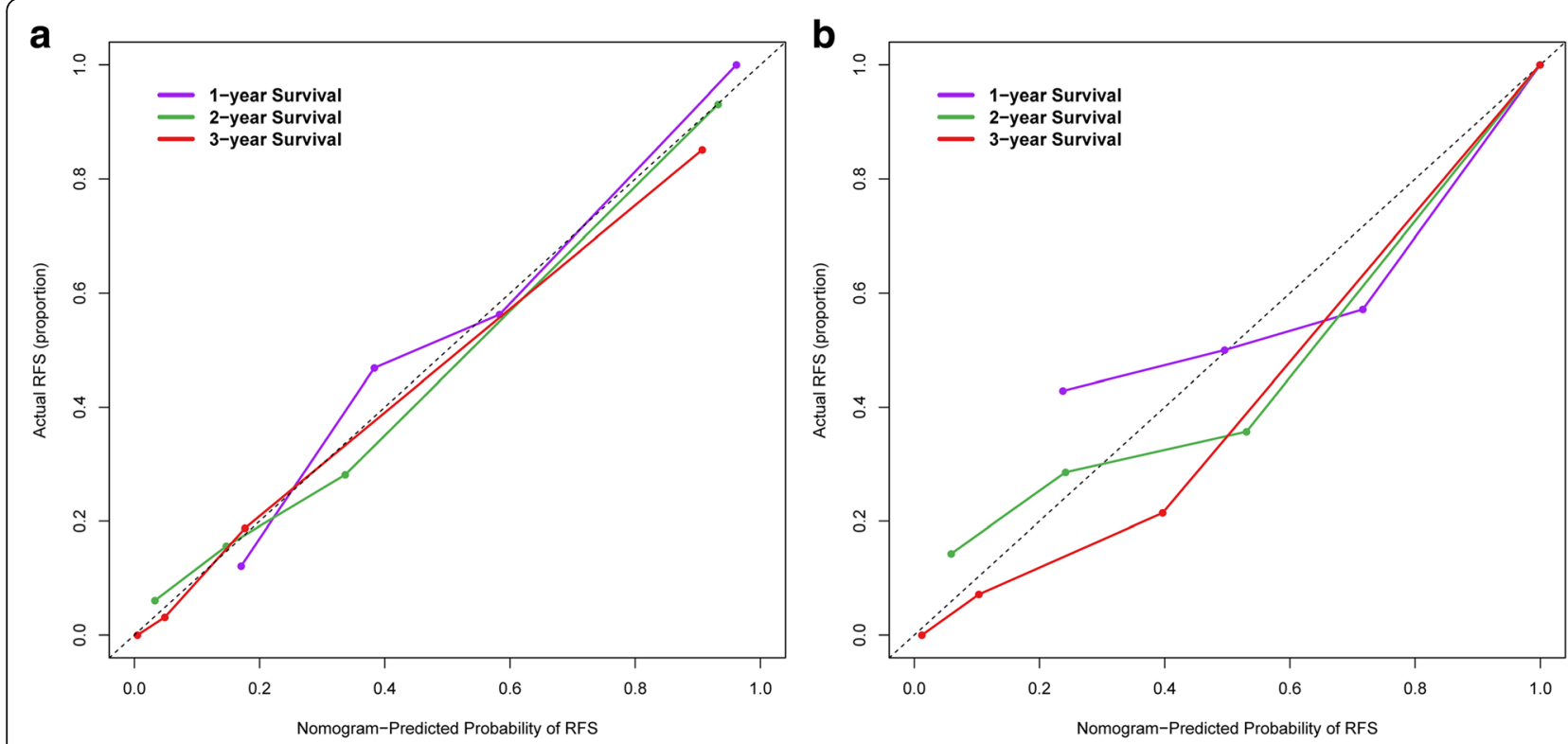

Fig. 5 Calibration curves of the combined nomogram in the (a) training and (b) validation datasets. The $y$-axis represents the actual recurrencefree survival (RFS). The $x$-axis represents the predicted RFS possibility. The diagonal dashed line indicates the ideal prediction by a perfect model

modified RECIST (mRECIST) standard) [45] was achieved in all patients, which is comparable to radical surgical resection [46]. Secondly, all patients were followed $>3$ years, which can be considered mid-term follow-up. Finally, the radiomics approach uses CECT images, which are routinely used for the diagnosis and follow-up of patients with HCC and widely available.

Furthermore, it has to be highlighted that radiomics results from specialized software instead of traditional imaging using the naked eye. In addition, radiomics captures more information about the tumor than percutaneous biopsy or histopathological examination of the surgical specimen, with little additional cost and good predictive outcomes [19]. Radiomics is still in its infancy, but a number of studies show promising results in a variety of cancers [25, 41-44]. Therefore, tumor recurrence prediction could be improved using radiomics nomograms. The present study improves the radiomics approach by including clinicopathological features in the final model, which has hardly been done before. One previous study suggested the use of the immunohistochemical markers CK19 and GPC3 for the prediction of HCC recurrence [47]. Future models could use a combination of radiomics, clinicopathological factors, and immunohistochemical characteristics of the HCC. In addition, the radiomics models should be directly compared with the available and widely used clinical models of HCC prognosis [14-16]. This has to be examined in the future.

Of course, the present study is not without limitations. This was a retrospective single center study spanning a long period of time. Therefore, several biases related to the treatment method and imaging could be present. In addition, the sample size was relatively small, external validation and larger datasets are needed to validate and refine our results.

\section{Conclusions}

The present study establishing a model for the prediction of HCC recurrence after curative ablation, that combined radiomics signature in the portal venous phase with clinicopathological features. This model could help stratify the patients in order to adopt the most appropriate follow-up and interventional strategy.

\section{Additional file}

Additional file 1: Table S1. Selected features for CT image in different phases. Table S2. Detailed information of the selected features in radiomics models. Table $\mathbf{S 3}$. Radiomics signature for $\mathrm{CT}$ image in different phases. (DOC 57 kb)

\section{Abbreviations}

AFP: alfa fetal protein; BCLC: Barcelona Clinic Liver Cancer; CECT: contrast enhanced computed tomography; CEMRI: contrast enhanced magnetic resonance imaging; Cryo-A: cryoablation; DICOM: digital imaging and communications in medicine; ECOG: Eastern Cooperative Oncology Group; HCC: hepatocellular Carcinoma; ICC: intra-class correlation coefficient; LASSO: least absolute shrinkage and selection operator; MRMRA: minimum redundancy maximum relevance algorithm; MWA: microwave ablation; PET$C T$ : Positron Emission Tomography-Computed Tomography;

RFA: radiofrequency ablation; RFS: recurrence free survival; ROI: Regions of interest; TACE: transarterial chemoembolization

Acknowledgements

None. 


\section{Funding}

National Natural Science Foundation of China (No.81371546,81527805, 81771924,61527807), Beijing Training Project For The Leading Talents in S \& T (No.Z141107001514002), Health Industry Special Scientific Research Project (No.201402019), Beijing Municipal Administration of Hospitals' Mission Plan (No.SML20150101), Beijing Scholar 2015 (No.160), Capital Health Research and Development of Special Fund (2018-2-2182), Beijing Municipal Science \& Technology Commission (No.Z181100001718070).

\section{Availability of data and materials}

Please contact authors for data requests.

\section{Authors' contributions}

ZCW and JT proposed the study. CWY and DSG performed research, analyzed the data and wrote the first draft. PZ, NH, YS, XZY, WFG collected the data, JWW, XHH, DD auxiliarily analyzed the data. JLF provided all the pathological data and reconfirmed the histologic grade. All authors contributed to the design and interpretation of the study and to further drafts. ZCW is the guarantor. All authors read and approved the final manuscript.

\section{Ethics approval and consent to participate}

The study protocol was approved by Institutional Review Board of our institution, the informed consent was not required.

\section{Consent for publication}

Not applicable.

\section{Competing interests}

The authors declare that they have no competing interests.

\section{Publisher's Note}

Springer Nature remains neutral with regard to jurisdictional claims in published maps and institutional affiliations.

\section{Author details}

'Department of Radiology, Beijing Friendship Hospital, Capital Medical University, No.95, Yong An Road, Xicheng District, Beijing 100050, China. ${ }^{2}$ Center of Interventional Oncology and Liver Diseases, Beijing Youan Hospital, Capital Medical University, Beijing 100069, China. ${ }^{3}$ Key Laboratory of Molecular Imaging, Institute of Automation, Chinese Academy of Sciences, No.95 Zhongguancun East Road, Haidian District, Beijing 100190, China. ${ }^{4}$ Center of Clinical Pathology, Beijing Youan Hospital, Capital Medical University, Beijing 100069, China. ${ }^{5}$ Beijing Advanced Innovation Center for Big Data-Based Precision Medicine, School of Medicine, Beihang University, Beijing 100191, China. ${ }^{6}$ Engineering Research Center of Molecular and Neuro Imaging of Ministry of Education, School of Life Science and Technology, Xidian University, Xi'an, Shanxi 710126, China. ${ }^{7}$ University of Chinese Academy of Sciences, Beijing 100049, China.

Received: 8 January 2019 Accepted: 2 April 2019

Published online: 26 April 2019

\section{References}

1. Forner A, Reig M, Bruix J. Hepatocellular carcinoma. Lancet. 2018;391:1301-14.

2. Zheng R, Qu C, Zhang S, et al. Liver cancer incidence and mortality in China: temporal trends and projections to 2030. Chin J Cancer Res. 2018:30(6):571-9.

3. Nault JC, Sutter O, Nahon P, Ganne-Carrie N, Seror O. Percutaneous treatment of hepatocellular carcinoma: state of the art and innovations. J Hepatol. 2017;68:783-97.

4. Liccioni A, Reig M, Bruix J. Treatment of hepatocellular carcinoma. Dig Dis. 2014;32(5):554-63.

5. Hasegawa K, Aoki T, Ishizawa T, et al. Comparison of the therapeutic outcomes between surgical resection and percutaneous ablation for smal hepatocellular carcinoma. Ann Surg Oncol. 2014;21(Suppl 3):S348-55.

6. Peng ZW, Zhang YJ, Chen MS, et al. Radiofrequency ablation with or without transcatheter arterial chemoembolization in the treatment of hepatocellular carcinoma: a prospective randomized trial. J Clin Oncol. 2013:31(4):426-32

7. Xie DY, Ren ZG, Zhou J, Fan J, Gao Q. Critical appraisal of Chinese 2017 guideline on the management of hepatocellular carcinoma. Hepatobiliary Surg Nutr. 2017:6(6):387-96.
8. Pompili M, Saviano A, de Matthaeis N, et al. Long-term effectiveness of resection and radiofrequency ablation for single hepatocellular carcinoma $</=3 \mathrm{~cm}$. Results of a multicenter Italian survey. J Hepatol. 2013;59(1):89-97.

9. Heimbach JK. Overview of the Updated AASLD Guidelines for the Management of HCC. Gastroenterol Hepatol (NY). 2017;13(12):751-753.

10. Heimbach JK, Kulik LM, Finn RS, et al. AASLD guidelines for the treatment of hepatocellular carcinoma. Hepatology. 2018;67(1):358-80.

11. Wu JC, Huang YH, Chau GY, et al. Risk factors for early and late recurrence in hepatitis B-related hepatocellular carcinoma. J Hepatol. 2009;51(5):890-7.

12. Portolani N, Coniglio A, Ghidoni S, et al. Early and late recurrence after liver resection for hepatocellular carcinoma: prognostic and therapeutic implications. Ann Surg. 2006;243(2):229-35.

13. Benson AB 3rd, D'Angelica Ml, Abbott DE, et al. NCCN guidelines insights: hepatobiliary cancers, version 1. 2017. J Natl Compr Cancer Netw. 2017;15(5):563-73.

14. Shim JH, Jun MJ, Han S, et al. Prognostic nomograms for prediction of recurrence and survival after curative liver resection for hepatocellular carcinoma. Ann Surg. 2015:261(5):939-46.

15. Cho CS, Gonen M, Shia J, et al. A novel prognostic nomogram is more accurate than conventional staging systems for predicting survival after resection of hepatocellular carcinoma. J Am Coll Surg. 2008;206(2):281-91.

16. Simons JP, Hill JS, Ng SC, et al. Perioperative mortality for management of hepatic neoplasm: a simple risk score. Ann Surg. 2009;250(6):929-34.

17. Segal $E_{\text {, Sirlin } C B}$, Ooi $C$, et al. Decoding global gene expression programs in liver cancer by noninvasive imaging. Nat Biotechnol. 2007;25(6):675-80.

18. Gillies RJ, Kinahan PE, Hricak H. Radiomics: images are more than pictures, they are data. Radiology. 2016;278(2):563-77.

19. Lambin P, Rios-Velazquez E, Leijenaar R, et al. Radiomics: extracting more information from medical images using advanced feature analysis. Eur J Cancer. 2012;48(4):441-6.

20. Ng F, Ganeshan B, Kozarski R, Miles KA, Goh V. Assessment of primary colorectal cancer heterogeneity by using whole-tumor texture analysis: contrast-enhanced CT texture as a biomarker of 5-year survival. Radiology. 2013;266(1):177-84.

21. Kumar V, Gu Y, Basu S, et al. Radiomics: the process and the challenges. Magn Reson Imaging. 2013;30(9):1234-48.

22. Yip SS, Aerts HJ. Applications and limitations of radiomics. Phys Med Biol. 2016;61(13):R150-66

23. Yip SSF, Liu Y, Parmar $C$, et al. Associations between radiologist-defined semantic and automatically computed radiomic features in non-small cell lung cancer. Sci Rep. 2017;7(1):3519.

24. Parekh V, Jacobs MA. Radiomics: a new application from established techniques. Expert Rev Precis Med Drug Dev. 2016;1(2):207-26.

25. Zhou Y, He L, Huang Y, et al. CT-based radiomics signature: a potential biomarker for preoperative prediction of early recurrence in hepatocellular carcinoma. Abdom Radiol (NY). 2017;42(6):1695-1704.

26. Bruix J, Sherman M. American Association for the Study of Liver Diseases. Management of hepatocellular carcinoma: an update. Hepatology. 2011:53(3):1020-2.

27. Aerts HJ, Velazquez ER, Leijenaar RT, et al. Decoding tumour phenotype by noninvasive imaging using a quantitative radiomics approach. Nat Commun .2014;4006.

28. Thibault G, Fertil B, Navarro C, et al. Shape and texture indexes application to cell nuclei classification. Int J Pattern Recognition Artificial Intel. 2013; 27(1):1357002 -1-23.

29. Galloway MM. Texture analysis using grey level run lengths. Nasa Sti/recon Technical Report .1974; N 75.

30. Amadasun M, King R. Textural features corresponding to textural properties. IEEE Transactions on Systems Man \& Cybernetics. 1989;9(5):1264-74.

31. Brown G, Pocock A, Zhao MJ, Luján M. Conditional likelihood maximisation: aunifying framework for information theoretic feature selection. J Mach Learn Res. 2012;13(1):27-66.

32. Parmar C, Grossmann P, Bussink J, Lambin P, Aerts HJ. Machine learning methods for quantitative radiomic biomarkers. Sci Rep. 2015;5:13087.

33. Sauerbrei $W$, Royston $P$, Binder $H$. Selection of important variables and determination of functional form for continuous predictors in multivariable model building. Stat Med. 2007;26(30):5512-28.

34. Tibshirani R. The lasso method for variable selection in the cox model. Stat Med. 1997;16(4):385-95.

35. Jr DWH, Lemeshow S, Sturdivant RX. Applied logistic regression, 3rd edition. Technometrics. 2013;34(3):358-9.

36. Balachandran VP, Gonen M, Smith JJ, De Matteo RP (2015) Nomograms in oncology: more than meets the eye. Lancet Oncol2015;16(4):e173-e180. 
37. Chen L, Sun J, Yang X. Radiofrequency ablation-combined multimodel therapies for hepatocellular carcinoma: current status. Cancer Lett. 2016;370(1):78-84.

38. Nakagawa S, Hayashi H, Nitta H, et al. Scoring system based on tumor markers and child-Pugh classification for HCC patients who underwent liver resection. Anticancer Res. 2015;35(4):2157-63.

39. Zhao WH, Ma ZM, Zhou XR, Feng YZ, Fang BS. Prediction of recurrence and prognosis in patients with hepatocellular carcinoma after resection by use of CLIP score. World J Gastroenterol. 2002;8(2):237-42.

40. Tateishi R, Shiina S, Yoshida H, et al. Prediction of recurrence of hepatocellular carcinoma after curative ablation using three tumor markers. Hepatology. 2006:44(6):1518-27.

41. Huang Y, Liu Z, He L, et al. Radiomics signature: a potential biomarker for the prediction of disease-free survival in early-stage (I or II) non-small cell lung cancer. Radiology. 2016;281(3):947-57.

42. Huang $\mathrm{YQ}$, Liang $\mathrm{CH}$, He L, et al. Development and validation of a radiomics nomogram for preoperative prediction of lymph node metastasis in colorectal cancer. J Clin Oncol. 2016;34(18):2157-64.

43. Coroller TP, Grossmann P, Hou Y, et al. CT-based radiomic signature predicts distant metastasis in lung adenocarcinoma. Radiother Oncol. 2015;114(3):345-50.

44. Liang $C$, Huang $Y$, He L, et al. The development and validation of a CTbased radiomics signature for the preoperative discrimination of stage $1-\|$ and stage III-IV colorectal cancer. Oncotarget. 2016;7(21):31401-12.

45. Lencioni R, Llovet JM. Modified RECIST (mRECIST) assessment for hepatocellular carcinoma. Semin Liver Dis. 2010;30(1):52-60

46. Xu Q, Kobayashi S, Ye X, Meng X. Comparison of hepatic resection and radiofrequency ablation for small hepatocellular carcinoma: a meta-analysis of 16, 103 patients. Sci Rep. 2014;4:7252.

47. Feng J, Zhu R, Chang C, et al. CK19 and Glypican 3 expression profiling in the prognostic indication for patients with HCC after surgical resection. PLoS One. 2016;11(3)::0151501.

Ready to submit your research? Choose BMC and benefit from:

- fast, convenient online submission

- thorough peer review by experienced researchers in your field

- rapid publication on acceptance

- support for research data, including large and complex data types

- gold Open Access which fosters wider collaboration and increased citations

- maximum visibility for your research: over $100 \mathrm{M}$ website views per year

At $\mathrm{BMC}$, research is always in progress.

Learn more biomedcentral.com/submissions 